\title{
Mathematical Modeling of Power Supply of an Autonomic Enterprise Based on Wind Power Plants
}

\author{
Valeri Telegin*, Anatoli Stepanov* \\ * Lipetsk state technical University, Russia
}

\begin{abstract}
Power supply systems for small businesses based on renewable energy sources are most often based on converting wind energy, solar energy and water energy. Calculating its effectiveness is a time-consuming task, requiring the processing of a large amount of data specific for the geographical location of power generating units. In the article the technique of computer modeling of work of a park of wind power plants (WPP) with the purpose of definition of an optimum parity of their parameters is considered.
\end{abstract}

Keywords: wind power plant, wind generator, computer modeling, wind energy.

\section{Introduction}

One of the problems of developing remote and previously underutilized regions is their power supply. Its solution is possible both through the construction of power transmission lines and through autonomous power supply, in particular, using renewable or alternative energy technologies: wind power plants (WPP), solar power plants (SPP) and small hydroelectric power plants (SHPP) $[1,2]$.

A characteristic feature of most alternative energy systems (AES) is the dependence of the generated power is the main indicator of their efficiency, on the dynamically changing amount of energy of the source. For WPP, this is the wind speed. Its average value depends on many factors, the main of which are the geographical position of the location of the WPP, the surrounding landscape, the time of the year, as well as data on possible fluctuations in the average wind speed.

The purpose of this work is to calculate the parameters that ensure the efficiency of the power supply system of a small enterprise based on the WPP, which includes wind generators (WG), batteries and the user of electricity.

\section{Energy Flows in a Closed Power Supply System}

The main indicator of the efficiency of the power supply system is the possibility of providing the consumer with electric power of not less than the required power [3]:

$P_{g}+P_{a} \geq P_{c} \cdot k_{r}$

where $P_{g}$ and $P_{a}$ - power generated and accumulated, $P_{c}-$ the power needed by the consumer, $k_{r}$ - is the safety coefficient.

Generated by the WPP park, the power in (1):

$P_{g}=0.5 \cdot N_{w t} \cdot \rho \cdot S_{r} \cdot V_{w}^{3} \cdot \eta_{w t} \cdot \eta_{p l}$ where $N_{w t}$ is the number of windmills; $\rho$ - density of air, $\mathrm{kg} / \mathrm{m}^{3} ; S_{r}$ - areas of wind-forest, $\mathrm{m}^{2} ; \eta_{w t}-$ к.П.д. ветродвигателя (ветроколесо, редуктор, генератор и др.); $\eta_{p l}$ - eff wind turbine (wind wheel, reducer, generator, etc.); $V_{w}$ - wind speed, $\mathrm{m} / \mathrm{s}$.

If the value of the generated energy $\left(P_{g}\right)$ exceeds that required by the consumer, the surplus energy is used to charge the batteries or is removed from the system by ballast resistance $\left(P_{b p}\right)$. Equations of the energy balance (3) - (6) in this case have the form:

$$
P_{a}=P_{g}-\frac{P_{c}}{k_{i}} \geq 0
$$

$\left\{\begin{array}{l}P_{a} \cdot t \leq D_{a} \cdot U_{a} \cdot k_{a} \\ I_{a}=\frac{P_{a}}{n_{a} \cdot U_{a}} \leq I_{c}\end{array} \Rightarrow\left\{\begin{array}{l}\bar{D}_{a}=D_{a}-\frac{P_{a} \cdot t}{U_{a}} k_{a} \\ P_{b p}=0,\end{array}\right.\right.$

$\left\{\begin{array}{l}P_{a} \cdot t \leq D_{a} \cdot U_{a} \cdot k_{a} \\ I_{a}=\frac{P_{a}}{n_{a} \cdot U_{a}}>I_{c}\end{array} \Rightarrow\left\{\begin{array}{l}\bar{D}_{a}=D_{a}-I_{c} \cdot t \cdot k_{a} \\ P_{b p}=P_{a}-I_{c} \cdot U_{a},\end{array}\right.\right.$

$\left\{\begin{array}{l}P_{a} \cdot t>D_{a} \cdot U_{a} \cdot k_{a} \\ I_{a}=\frac{P_{a}}{n_{a} \cdot U_{a}} \leq I_{c}\end{array} \Rightarrow\left\{\begin{array}{l}\bar{D}_{a}=0 \\ P_{b p}=P_{a}-\frac{D_{a} \cdot U_{a}}{k_{a} \cdot t}\end{array}\right.\right.$

$\left\{\begin{array}{l}P_{a} \cdot t>D_{a} \cdot U_{a} \cdot k_{a} \\ I_{a}=\frac{P_{a}}{n_{a} \cdot U_{a}}>I_{c}\end{array} \Rightarrow\left\{\begin{array}{l}\bar{D}_{a}=D_{a}-I_{c} \cdot t \cdot k_{a} \\ P_{b p}=\left(P_{a}-I_{c} \cdot U_{a}\right)\end{array}\right.\right.$

where $n_{a}$ - the number of batteries connected in parallel; $U_{a}$, B and $I_{a}, \mathrm{~A}-$ charge voltage and current of one accumulator battery, $k_{a}<1$ - eff battery; $D_{a}$ and $\bar{D}_{a}, \mathrm{~A} \cdot \mathrm{q}-$ initial and final values of the "free" capacity of the accumulation system $P_{a}, \mathrm{Vt}-$ surplus power, part of which can be accumulated or scattered by the bal- 
last load, $P_{b p}, \mathrm{Vt}-$ the power used by the ballast load, $k_{i}<1$-eff inverter.

The amount of energy that can be saved at the current time (t) is determined by two parameters: the "free" capacity of the storage system $\left(D_{a}\right)$ and the maximum value of the charging current $\left(I_{c}\right)$. If the battery capacity is $C_{a}$, then

$$
C_{a} \cdot\left(1-k_{d c}\right) \geq D_{a},
$$

where $k_{d c}-$ is the acceptable battery discharge factor. Usually $k_{d c} \approx$ 0.2 .

If the value of generated energy $\left(P_{g}\right)$ is less than what is necessary for the consumer, then its shortage is compensated by the discharge of batteries. The equations of the energy balance (7) - (8) in this case have the form:

$$
\begin{aligned}
& P_{a}=P_{g}-\frac{P_{c}}{k_{i}}<0 \\
& \left\{\begin{array} { l } 
{ | P _ { a } | \cdot t \leq [ ( 1 - k _ { d c } ) \cdot C _ { a } - D _ { a } ] \cdot U _ { a } } \\
{ I _ { a } = \frac { | P _ { a } | } { n _ { a } \cdot U _ { a } } \leq I _ { \operatorname { m a x } d c } }
\end{array} \Rightarrow \left\{\begin{array}{l}
\bar{D}_{a}=D_{a}+\frac{\left|P_{a}\right| \cdot t}{U_{a}} A_{d c}\left(I_{a}\right) \\
\bar{C}_{a}=\left(1-k_{d c}\right) \cdot C_{a}-\bar{D}_{a}>0,
\end{array}\right.\right.
\end{aligned}
$$

where $I_{\max d o}, \mathrm{~A}-\mathrm{A}$ is the maximum permissible battery discharge current, and $A_{d c}\left(I_{\mathrm{a}}\right)$ - is some correction function of the discharge current in the accumulation system circuit, the value of which is $\geq$ ${ }_{1}, \bar{C}_{a}$ the actual capacity of the battery. The value of the parameters $I_{\max d c}$ and $\bar{C}_{a}$ is determine the operability of the power supply system of the consumer. By Telegin V.V. the following analytical expression for the corrective function is proposed:

$$
\begin{cases}A_{d c}=a \cdot e^{I_{d c}^{k}}+b & I_{d c}>\frac{C_{10}}{T_{10}} \\ A_{d c}=B\left(I_{d c}\right) & I_{d c} \leq \frac{C_{10}}{T_{10}}, \\ A_{d c}=1 & \frac{C_{10}}{T_{10}}<I_{d c} \leq \frac{C_{20}}{T_{20}},\end{cases}
$$

\section{Mathematical Model of Power Supply Sys- tem}

The generated power (2) is in general a function of time, the values of which are available at discrete points $t_{i}$ given through constant time intervals:

$\Delta T=t_{i+1}-t_{i}=$ const

Assuming that the function $P_{g}(t)$ changes linearly from the value of $P_{1}$ to $P_{2}$ and the power consumption $P_{c}$ is constant, we have 4 variants of expressions: (11) - (14) for calculating the excess $W_{e x t}$ and the missing $W_{n s}$ energy with the following four possible variants of the relations between the values of $P_{1}, P_{2}$ and $P_{c}$ :

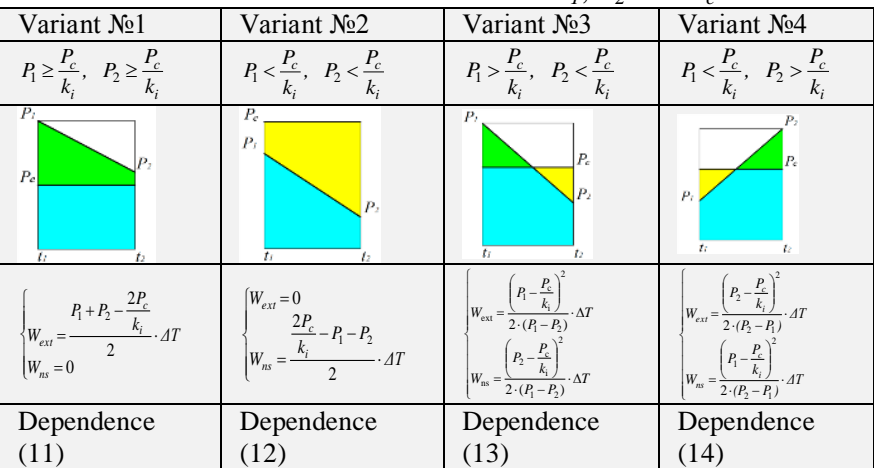

Thus, in the time interval $\left[t_{i}, t_{i+1}\right] \quad i=0,1,2 \ldots m$, the power value, redundant $\Delta W_{i} \geq 0$ or the missing $\Delta W_{i}<0$,in accordance with the dependences (10) - (14), is:

$$
\Delta W_{i}=W_{e x t}-W_{n s}
$$

where $m$ is the sequence number of the time interval.

If $\Delta W_{i} \geq 0$, then part of the excess energy $\Delta D_{a} \cdot \Delta T_{i}$ can be accumulated:

$$
\Delta D_{i}=D_{\mathrm{i}}-\bar{D}_{\mathrm{i}}
$$

and the rest is scattered. At the same time, the energy balance of the system is described by the following interrelation:

$a=\frac{C_{n}}{C_{10} C_{5}} \cdot \frac{C_{10}-C_{5}}{e^{\left(0.2 C_{5}\right)^{k}}-e^{\left(0.1 C_{10}\right)^{k}}}, \quad b=\frac{C_{n}}{C_{10} C_{5}} \cdot \frac{C_{5} \cdot e^{\left(0.2 C_{5}\right)^{k}}-C_{10} \cdot e^{\left(0.1 C_{10}\right)^{k}}}{e^{\left(0.2 C_{5}\right)^{k}}-e^{\left(0.1 C_{10}\right)^{k}}} \Delta D_{i}+P_{b p i}=\frac{\Delta W_{i}}{\Delta T}$

$B\left(I_{d c}\right)=\frac{T_{20} \cdot\left(C_{n}-C_{1}\right) \cdot I_{d c}-C_{n}^{2}+C_{1}^{2}}{\left(2 C_{1}-C_{n}\right) \cdot C_{1}}$.

The value of the coefficient $k$ for this particular battery can be obtained from the numerical solution of the equation:

$$
a \cdot e^{\left(\frac{C_{1}}{T_{1}}\right)^{k}}+b=\frac{C_{n}}{C_{10}} \text {. }
$$

In the expression (9) $C_{n}, C_{1}, C_{10}, C_{5}$, - the capacity of the accumulator (A·ч) is nominal, for 1,10 and 5 -hour discharges, $T_{10}, T_{20}$ is the charge (discharge) time (h) 10 and 20 hours, accordingly, $I_{d c}$ - is the discharge current (A), $k$ - is the dimensionless coefficient. Function $B\left(I_{d c}\right)$ will be equal to one for most battery models, for which $C_{n}=C_{10}$. If the nominal capacity of the battery corresponds to its twenty-hour discharge $\left(C_{20}\right)$, it is assumed that in the interval $\left[C_{20}, C_{10}\right] A_{d c}=B$.

where $D_{a}, \bar{D}_{a}, P_{b p i}$ are determined from equations (3) - (9) with allowance for the following equalities:

$P_{a}=\frac{\Delta W_{i}}{\Delta T}, \quad P_{b p i}=P_{b p}, \quad \Delta T=t$.

If $\Delta W_{i}<0$, the missing part of the energy $\Delta D_{a} \cdot \Delta T_{i}$ must be replenished from the accumulation system. The equation of the energy balance of the system in this case:

$$
\frac{\Delta W_{i}}{\Delta T}=\Delta D_{\mathrm{i}}
$$

where $\Delta D_{a}$ can be found from Eqs. (8) with allowance for (18).

Figure 1 shows the scheme of operation of the accumulation system in the modes of accumulation (charge) and energy release (discharge). 


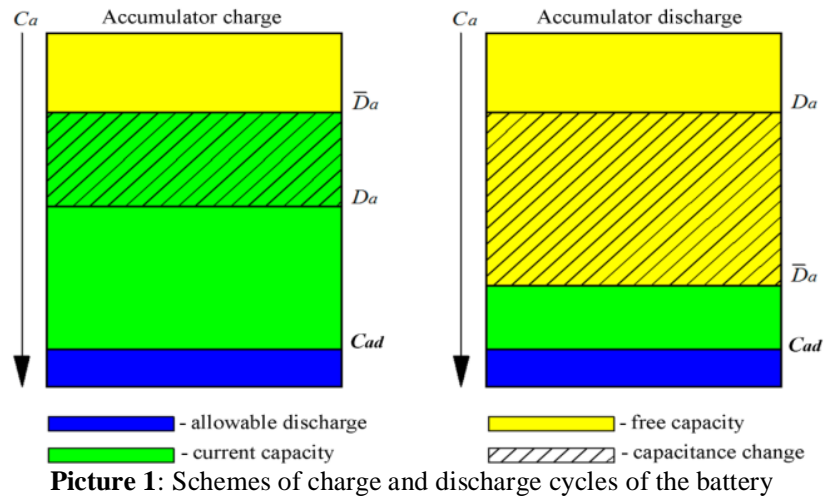

Relations (1) - (19) represent a mathematical model of the system of autonomous power supply to consumers based on alternative energy sources. It should be borne in mind that the parameters of this model, in addition to those explicitly indicated in expressions (1) - (19), are also the characteristics of 3D models [4] of elements of WPP, and its cost too [5]. The solution of the modeling problem involves the implementation of a sequence of mathematical calculations, for $\mathrm{m}$ time points $\left(t_{1}, t_{2}, \ldots, t_{m}\right)$, which divides the time interval $T$ into an $m-1$ interval.

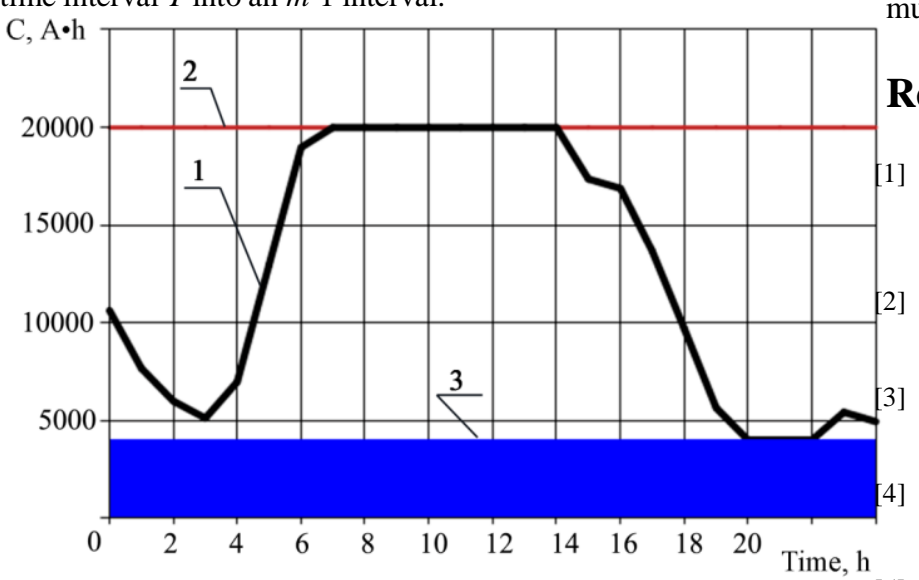

Picture 2: Changing battery capacity: 1 - current, 2 - nominal, 3 - mini- [5] mum (maximum permissible discharge)

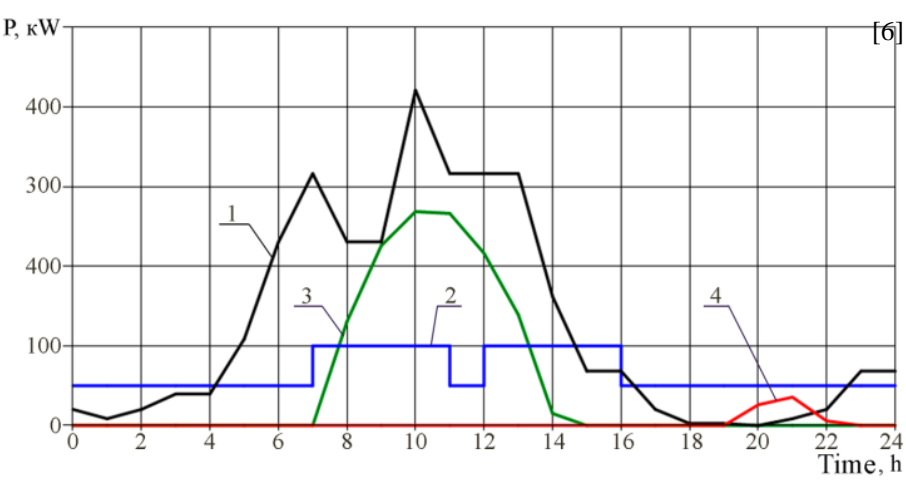

Picture 3: Energy balance in the system of autonomous power supply to the consumer: 1 - generated, 2 - consumed, 3 - redundant, 4 - missing.

The results of testing the mathematical model are shown in Pictures 2,3 .

Parameters of the model under test:

1. Number of WPP - 25. The height of the mast is $-20 \mathrm{~m}$, the diameter of the wind wheel is $10 \mathrm{~m}$. Coefficient of efficiency: wind wheel -0.46 , reducer -0.85 , generator -0.8 . The minimum permissible wind speed required for WPP operation is $2 \mathrm{~m} / \mathrm{s}$.

2. Accumulators - 100 pcs. Model GX 12-200. The coefficient of efficiency is 0.93 .

3. Transmission and energy conversion systems. The efficiency of the transmission line is 0.94 , the controller is 0.94 , the inverter is 0.94 .
4. Consumed power from 100 to $50 \mathrm{~kW}$ - dependence 2 in Picture 3.

The wind speed is equal to the wind speed measured at the meteorological station of the city of Lipetsk, at an altitude of 10 meters on May 17, 2012.

\section{Conclusion}

The proposed mathematical model makes it possible to evaluate the effectiveness of using renewable energy sources for electricity supply to consumers. However, obtaining reliable results for a long period of time (from several months to several years) requires the use of statistical data on the wind speed at a given point collected over several years. The use of the mathematical model is associated with a large amount of calculations, the implementation of which can be performed with the help of specially developed software [5, 6].

The mathematical model considered in the article forms the basis of the software complex "Alternative Energy Technology" (AET), which allows to calculate the parameters of the electro-generating complex, including wind power plants, solar panels and hydraulic turbines, which ensure the extremum of the given criterion: maximum power, minimum cost, and others [6].

\section{References}

Shpiganovich, A.N., Telegin V.V., Energy saving with use of autonomous sources based on alternative energy technologies, Journal higher educational institutions of the Chernozem region. (2011), No 4 (26), pp. 16-21.

Telegin, V.V., On the issue of experience world in use of renewable energy sources, Proceedings of the V International Conference "Scientific Potential 21st Century". (2011), Vol. 1, P. 178-181. Telegin, V.V., Kozlov, A.M., Sakalo, V.I. Solid Modeling and Dynamic Analysis of Mechanisms of Press-forging Machines, Procedia Engineering. (2017), P. 1258-1263.

Telegin V.V., Optimization of structure and parameters of autonomous electrogenerating complexes, Fundamental research. (2013). Vol. 8-2. P. 312-317.

Telegin V.V., Computer modeling of efficiency using alternative energy systems, Natural and technical sciences. (2012), No. 5 (61), pp. 309-312.

The certificate № 2012660892 Russian Federation. Certificate of state registration computer program. Alternative Energy Technologies (TAE) / V.V. Telegin; applicant and copyright holder Telegin Valeriy Viktorovich (RU). - №2012660892; claimed. 10/22/12; publ. 30.11.12, Register of Computer Programs 